\title{
Statistical Analysis of Chronic Subdural Hematoma [in 309 Adult Cases
}

\author{
Kimiyoshi Hirakawa, M.D., Keizo Hashizume, M.D., \\ Tokuro Fuchinoue, M.D., Hiroshi Takahashi, M.D., \\ Kazuhiro Nomura, M.D., Masao Matsutani, M.D., \\ and Keiji SANO, M.D.
}

Chronic subdural hematoma is a common disease. Occasionally, however, it is so puzzling to make a diagnosis clearly and immediately, that the delay of the management may occur. So the purpose of this paper is to clarify the general picture of chronic subdural hematoma from a statistical analysis in 309 adult cases. Through this survey the role of trauma as the triggering factor is to be checked and the signs and symptoms will be summarized and divided into groups according to clinical features, which will make the diagnosis easy and useful to dissolve the pitfall. Pathophysiology and dynamics of the capsule of the hematoma are investigated in some cases, and the dysfunction of the liver is also inquired. Follow-up study was performed, which will contribute to the selection of the operative method.

\section{Materials and Methods}

This report was based on a study of 309 out of 351 patients with chronic subdural hematoma who were admitted to the Department of Neurosurgery, University of Tokyo and its affiliated hospitals between 1948 and 1972. Age distribution is shown in Fig. 1, in which there are two peaks, elderly side and infancy side. Occurence in the preschool childhood being so rare, and if any, signs and symptoms being so similar to those of the adult, the authors regard as the adult type above 5 years of age, though in this report minimum age was 7 and maximal age was 79 years old. The peak was in the sixth decade. Important was the sex difference; $91 \%$ was male and $9 \%$ was female.

Chronic subdural hematoma in this report is defined as follows. One is a case whose onset of the disease is 3 weeks after the preceeding trauma, and another one is the encapsulated hematoma whose clinical pictures are similar to those of the former, but their onset of the disease is less than 3 weeks after the trauma or they have no history of trauma.

\section{Statistical Analysis}

History of Trauma

History of trauma preceeding the onset of the disease was noted in 276 cases $(89 \%)$, in which were the traffic accidents $47 \%$, ordinary life $35 \%$, sport accidents $10 \%$, industry accidents $3 \%$, and so on (Table 1 ). In sport accidents 9 cases were

Department of Neurosurgery, Faculty of Medicine, University of Tokyo 


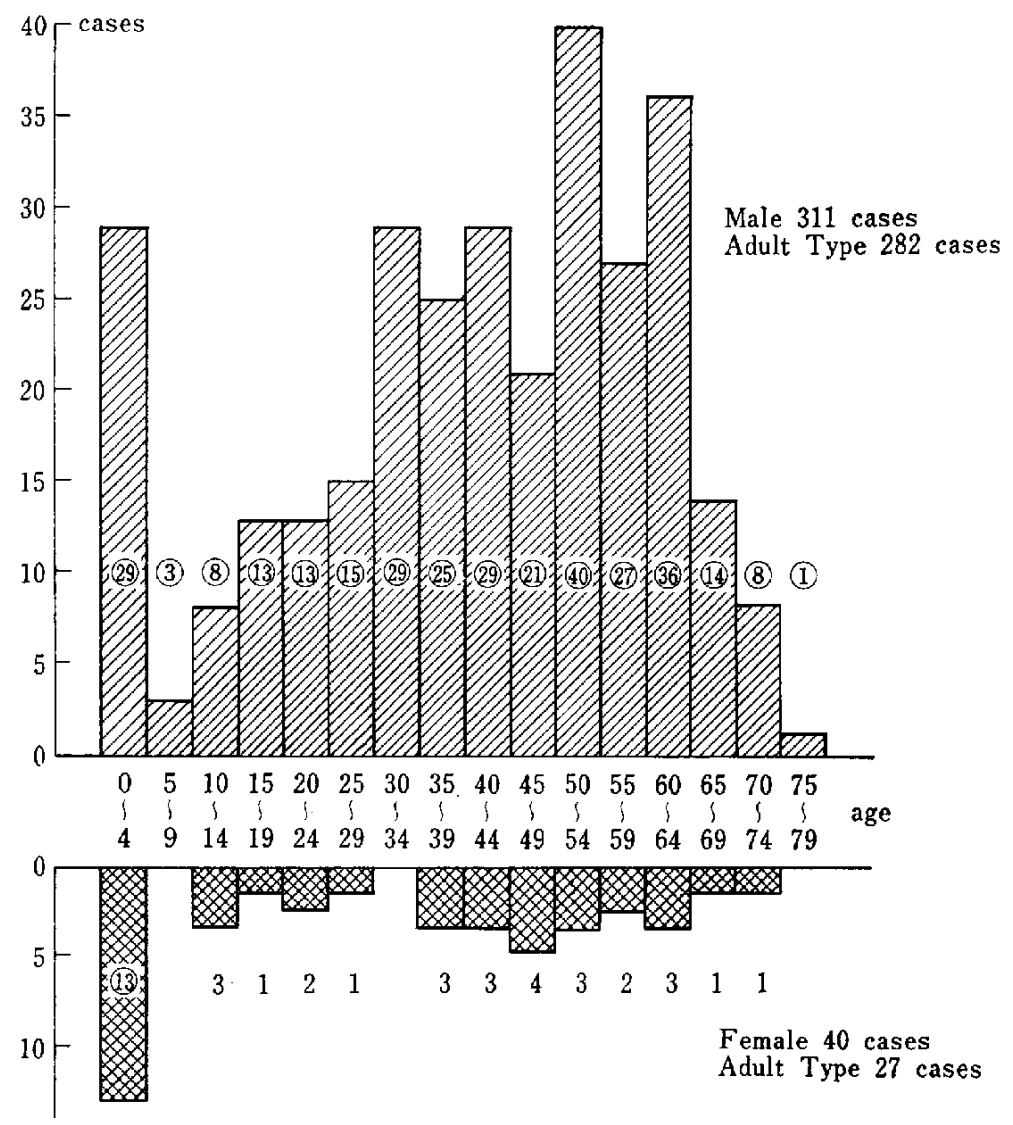

Fig. 1 Age distribution of 351 cases of chronic subdural hematoma

caused by Judo. They fell down on their back. Mode of the traumas was surveyed in 231 well-defined cases (Table 2). Tumbling down were $43 \%$, falling down $27 \%$, collision $17 \%$, run against $13 \%$, struck $6 \%$, and falling objects $5 \%$. The location of the impacts was also surveyed in 208 well-defined cases (Table 3). Face and/or frontal area was $47 \%$, occipital area $30 \%$, parietal area $15 \%$, and temporal area $13 \%$. These values were compared with those of the 1771 trauma cases in the out-patient clinic as a control. They were $30 \%, 15 \%, 15 \%, 19 \%$ respectively and whiplash injuries were encountered in $5 \%$. So, the sagittaly directed blows were dominant in the hematoma group. The duration of the initial unconsciousness was surveyed as an index of the strength of impact (Table 4). In 258 cases patients without unconsciousness were $45 \%$, within 5 minutes $21 \%$, within 30 minutes $15 \%$, within 1 hour $4 \%$, within 6 hours $4 \%$, and more than 6 hours $7 \%$. Skull fractures were noticed in 19 cases $(7 \%)$.

Overwhelming numbers of the patients have the history of head injury, of which the patients have good memory even if consciousness disturbance had not occurred. 
Table 1 Cause of traumas

\begin{tabular}{lrl}
\hline traffic accident & 130 & $(47.1 \%)$ \\
ordinary life & 97 & $(35.1 \%)$ \\
sport accedent & 27 & $(9.8 \%)$ \\
industry accident & 9 & $(3.3 \%)$ \\
others & 13 & \\
no history of trauma & 33 & $(10.7 \%)$
\end{tabular}

Table 2 Mode of traumas

\begin{tabular}{lrr}
\hline tumbling down & 100 & $(43.3 \%)$ \\
falling down & 38 & $(16.5 \%)$ \\
collision & 38 & $(16.5 \%)$ \\
run against & 30 & $(13.0 \%)$ \\
struck & 14 & $(6.1 \%)$ \\
falling object & 11 & $(4.8 \%)$
\end{tabular}

Table 3 Location of impacts

\begin{tabular}{lrll}
\hline & chronic hematoma & control \\
face \& frontal & 97 & $(46.6 \%)$ & $(29.8 \%)$ \\
occipital & 63 & $(30.3 \%)$ & $(15.0 \%)$ \\
parietal & 31 & $(14.9 \%)$ & $(15.0 \%)$ \\
temporal & 26 & $(12.5 \%)$ & $(18.7 \%)$ \\
whiplash & 0 & $(0 \%)$ & $(4.5 \%)$
\end{tabular}

Table 4 Duration of initial unconsciousness

\begin{tabular}{lrl}
\hline none & 116 & $(45.0 \%)$ \\
-5 minutes & 54 & $(20.9 \%)$ \\
-30 minutes & 39 & $(15.1 \%)$ \\
-1 hour & 10 & $(3.9 \%)$ \\
-6 hours & 21 & $(8.1 \%)$ \\
6 hours - & 18 & $(7.0 \%)$
\end{tabular}

Recently chronic subdural hematomas after whiplash injuries were reported ${ }^{4)}$, though the authors have not experienced such a case. These facts, however, together with sagittaly directed blow being so high are significant keys to decide the effect of physical force over the brain. Trivial blow may sometimes has enough mechanical power for shearing damage to the surface of the brain and the bridging veins. So the effect of trauma should not be underestimated.

\section{Free Interval}

The interval from the trauma to the onset of the disease was surveyed (Fig. 2). The cases continuing from the trauma or beginning within 3 weeks were $18 \%$, within 1 month $13 \%, 2$ months $42 \%, 3$ months $17 \%, 6$ months $8 \%$, and more than 6 months $3 \%$. The interval between the trauma and the admission or the operation were also investigated. The patients operated within 3 weeks were $2 \%$, within 1 month $5 \%, 2$ months $22 \%, 3$ months $35 \%, 6$ months $30 \%$ and more than 6 months $6 \%$.

Signs and Symptoms

There were no pathognomonic findings of chronic subdural hematoma. Headache 

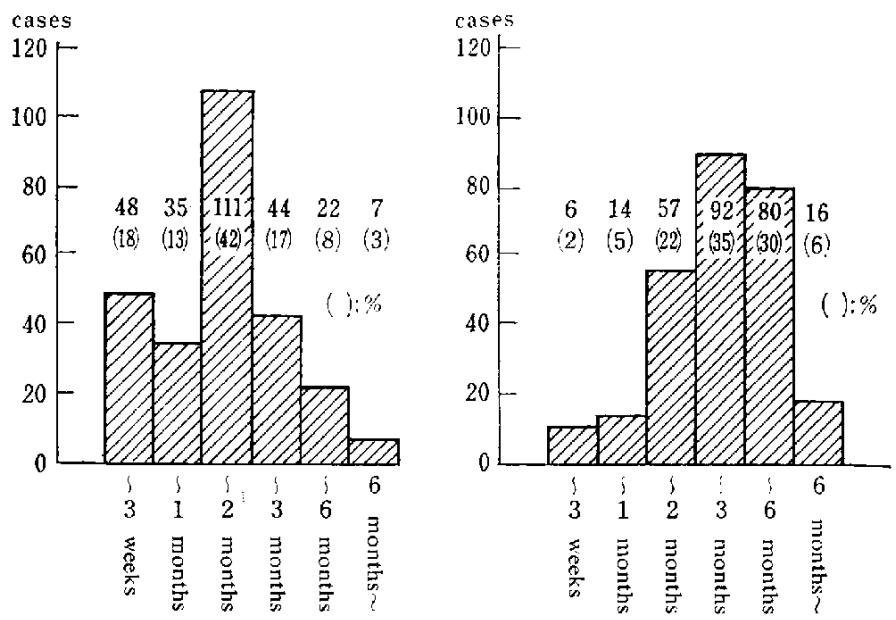

Fig. 2 Time interval from the trauma to the onset of the disease (left) and from the trauma to the operation (right).

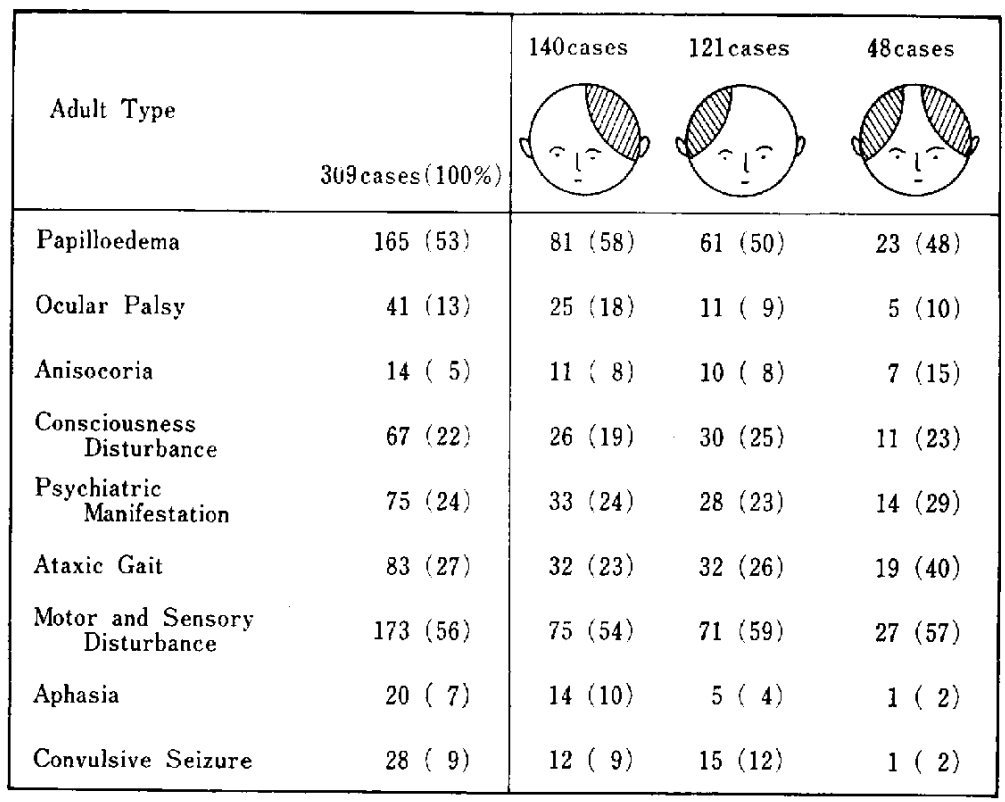

Table 5 Symptomatology of chronic subdural hematoma 

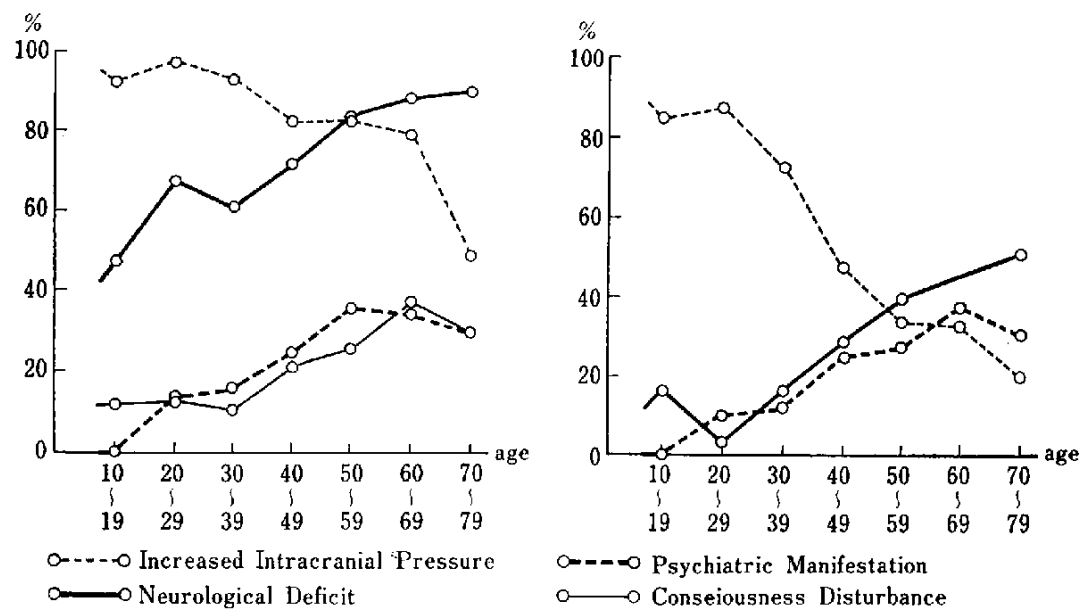

Fig. 3 Appearance rate of three cardinal symptoms of chronic subdural hematoma by age (upper figure), and that of the most prominent symptom of the three (lower figure).

and vomiting were seen in almost all of the patients. Symptoms elicited from 309 cases are shown in Table 5. Motor and sensory disturbances were seen most frequently in $56 \%$, in which laterality of reflex was included. Trivial weakness of movements of legs such as dragging and slipping, which will not be recognized by non-professional eyes, should be paid attention to for diagnosis. Sometimes, they were misunderstood as the brain infarction. Ataxic gaits were seen in $27 \%$ and were often initial symptoms of the disease. Awkward walking, staggering gait, and diffi culties in standing up were all encountered in ataxic gait, which were usually mistaken as the cerebellar involvement. Psychiatric manifestations were seen in $24 \%$, in which loss of recent memory, decreased abilities of calculation, and character changes were included. They were likely to be believed as the senile dementias. Consciousness disturbances were seen in $22 \%$ which will be mentioned later. Extraocular paresis, convulsive seizures, and anisocoria were also noticed. Papilloedemas were seen in $53 \%$.

The appearance rates of these symptoms were checked by the sides of the hematomas. But there was no significant difference among the left side hematomas ( 140 cases), the right side hematomas (121 cases), and bilateral hematomas (48 cases), except for appearance rate of ataxic gait the percentages of which are $23,26,40$ respectively.

These symptoms can be arranged in three main groups by their cardinal symptoms; symptoms showing increased intracranial pressure, neurological deficits, and psychiatric manifestations (including consciousness disturbances). They were checked by age (Fig. 3). Increased intracranial pressure was seen in any age groups except for the oldest one, while neurological deficit was seen about half in the younger age groups and were seen frequently in the elderly groups. Psychiatric manifestations were scarcely seen in younger groups and were prominent as age went higher. 
Table 6 Onset and course

\begin{tabular}{lrl}
\hline remission & 90 & $(29.1 \%)$ \\
slow progress & 138 & $(44.7 \%)$ \\
acute exacerbation & 48 & $(15.5 \%)$ \\
sudden onset $\&$ acute & 31 & $(10.0 \%)$ \\
loss of consciousness & &
\end{tabular}

Table 7 Classification of clinical picture

\begin{tabular}{lr}
\hline 1. cardinal symptoms consisting of & \\
increased intracranial pressure & 162 \\
neurological deficit & 80 \\
psychiatric manifestation & 67 \\
2. atypical symptoms consisting of & \\
vascular involvement & 7 \\
meningitis & 6 \\
epilepsy & 1 \\
muscle rigidity & 2 \\
blood dyscrasia & 1
\end{tabular}

\section{Course of Symptoms}

Generally speaking the course of symptoms of the chronic subdural hematomas is slowly progressive with some remmision. Some cases, however, during their chronic course of discomfort, show sudden headache and vomiting, become unsteady in walking, and then fall into consciousness disturbances within a few days. Some cases having no trouble at all suddenly fell into coma in a few days. The incidence of each types were $29 \%$ in purely remission type, $45 \%$ in slowly progressing type, $16 \%$ in acute exacerbation type, and $10 \%$ in sudden onset and acute course type. One forth of all cases may fall into unconsciousness (Table 6). In other words chronic subdural hematomas should be taken into acount in differentiation of consciousness disturbances.

Classification by Prominent Symptoms

Chronic subdural hematomas were classified by the prominent signs and symptoms in order to make the diagnosis easy. Typically they were classified into three types as described above; type of increased intracranial pressure 162 cases, neurological deficit 80 cases, and psychiatric manifestation 67 cases. But among these cases there were atypical cases in which misleading of the diagnosis were probable from situation of onset, clinical course etc. (Table 7). There were 5 atypical groups. Seven cases of vascular accident type were misleading to subarachnoid hemorrhage by their sudden onset of the disease and nuchal rigidity. Six cases of meningitis or encephalitis type manifested fever accompanied by nuchal rigidity. One case of epileptic type had repeated attacks of generalized convulsion. Two cases of rigidity type were misdiagnosed as Parkinsonism because of their muscle rigidity and pulsion. One case of the blood dyscrasia was macroglobulinemia. Exsistence of these atypical cases should always be taken into consideration in differential diagnosis.

Localization of Hematomas

Diagnosis of chronic subdural hematoma is easy once suspection is laid. Carotid 


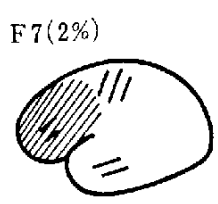

FPT $72(25 \%)$

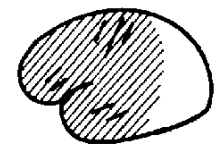

FT $12(4 \%)$

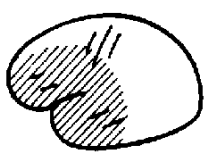

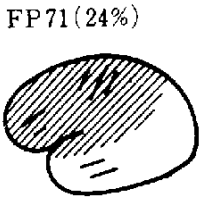

FPTO $87(30 \%)$

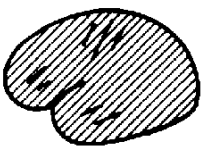

PT $8(3 \%)$

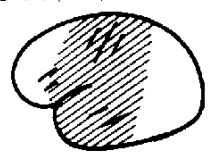

$\operatorname{FPO} 13(4 \%)$

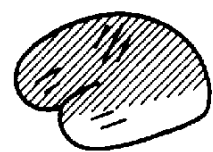

$\mathrm{PO} 1(0.3 \%)$

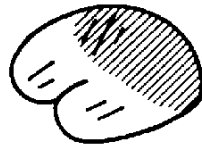

$\mathrm{P} 17(6 \%)$

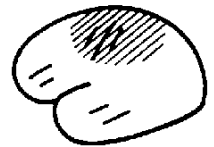

Fig. 4 Localization of the hematomas

angiography reveals avascular area and makes sure of the diagnosis. But to show the avascular area is not necessarily the sole proof of chronic subdural hematoma,because subdural hemorrhage from malignant glioma may occur. To know the localization of the hematoma is therefore inevitable to eliminate misdiagnosis (Fig. 4). Hematoma covering all of the hemisphere were seen in $30 \%$, while covering fronto-parieto-temporal area were $25 \%$, fronto-parietal area $24 \%$ and so on. Any way hematomas will be found $88 \%$ in frontal region, $93 \%$ in the parietal region, and were scarcely found in the occipital region. So that localization of avascular area will be a reliable tool to eliminate the hematoma.

Configuration of Avascular Area

On the antero-posterior view of carotid angiography variations of configuration of avascular area were noticed. They are divided into three types after the shape of lens, convexo-convex type (typical figure), plano-convex type (half moon shape), and convexo-concave type (crescent shape). From experiences of spontaneous recovery of chronic subdural hematomas which were already reported, the transformation of avascular area was closely related to expansion and regression of the hematoma. ${ }^{2), 3)}$ Avascular areas of convexo-concave type in this series were seen in the cases of very early stages after the trauma, in the operations where the hematoma was almost absorbing, in the cases where complicaions were disappearing without operation, and in the cases of calcified hematoma. It was not experienced in the cases showing sudden loss of consciousness. Above mentioned facts with other supports have suggested that chronic subdural hematoma has a life cycle concerning expansion and regression, which is classified into 4 stages; the stage of encapsulation, expansion, regression, and termination.

Dynamics of the Capsule

Hematoma or the capsule of the hematoma is a kind of inflammatory process or reactive product to subdural hemorrhage. Therefore, dynamics of the capsule is inter- 


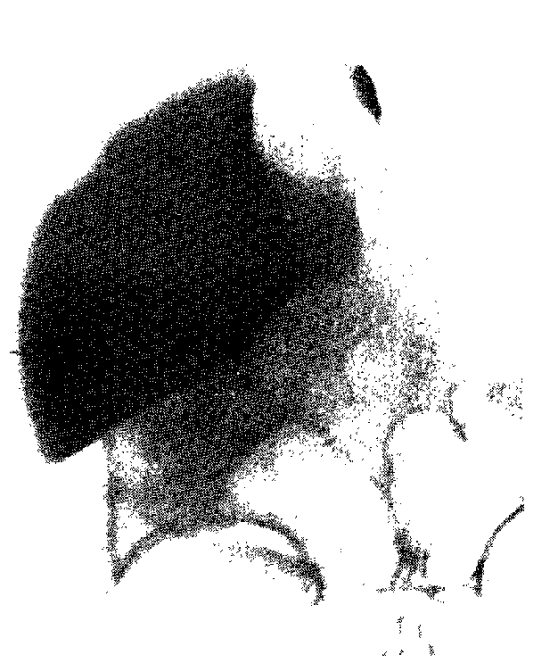

(A)

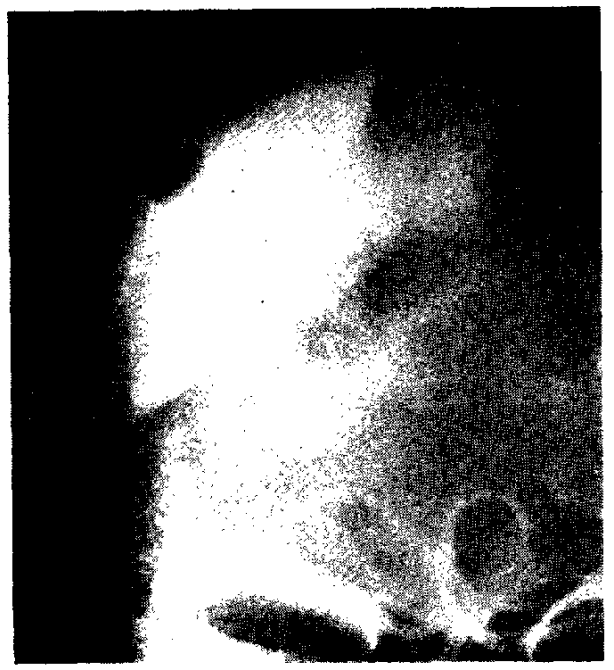

(B)

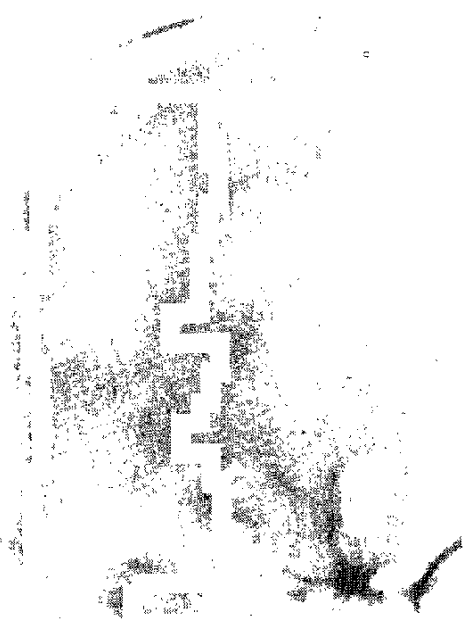

(C)

Fig. 5 Disappearance of Conray injected intraluminally.

A) immediate after injection, B) 6 hours later, and C) 22 hours later.

esting to note. It was proved that hemorrhages from the capsule into the lumen of the hematoma occurred repeatedly ${ }^{3)}$, while it should happen that contents (blood) of the hematoma is absorbed through the capsule.

To confirm the dynamics of absorption $3 \mathrm{ml}$ of water soluble contrast medium, Iothalamate (Conray), was injected into the lumen in several cases during the oper- 


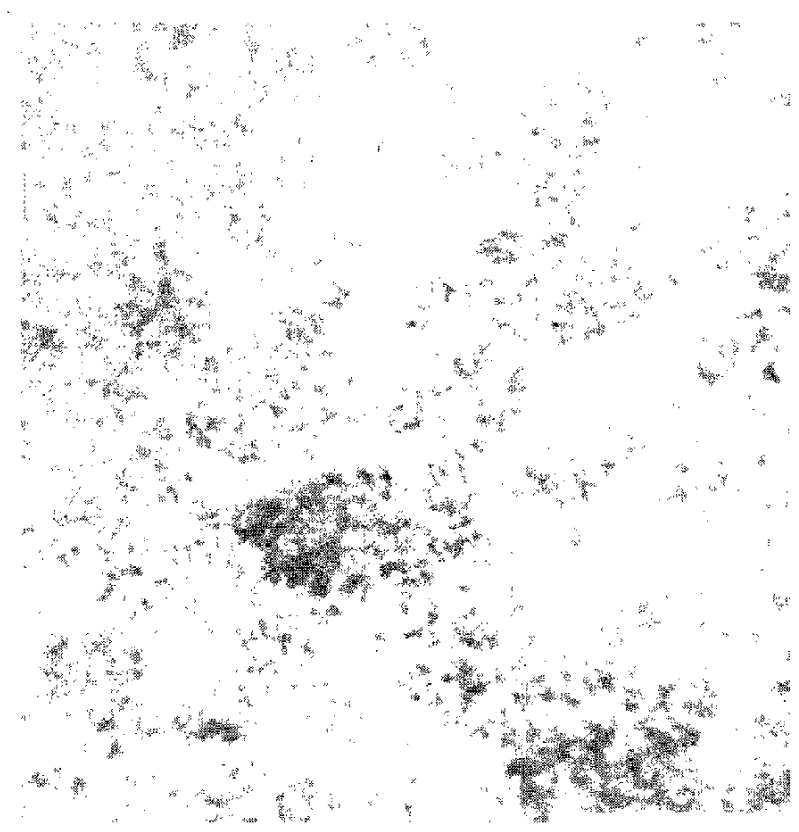

Fig. 6 Autradiogram of the capsule. Three hours after intraluminal injection of $196 \mathrm{Yb}$ (Hematoxylin stain, $\times 400$ )

ation. The density of the contrast medium in the lumen was checked by taking the $\mathrm{X}$-ray films successively. Excretion to the urine was also checked by X-ray, and it was found that Conray was detected in the urine within a few hours. The density of Conray in the hematoma decreased definitely after 22 hours (Fig. 5).

Another method to confirm the dynamics of the absorption was to utilize radioisotopes. $700 \mu \mathrm{c}$ of ${ }^{196} \mathrm{Yb}$ was by chance injected into the lumen of a hematoma, a case having a rather longer course. A piece of the capsule was removed 3 hours later to be examined by microscopic autoradiography. The specimen was cut by a cryostat for $4 \mu$ thick and incubated for 46 days. Background activities were so high, but the grains were seen scattered deeply in the capsule, especially around the vessels (Fig. 6). Therefore, the exchange of blood or substances through the capsule will be done actively according to the stage of the hematomas.

Capillaries and giant capillaries of sinusoidal vascular channel layer of the capsule were investigated by electronmicroscope. Capillaries with a diameter of 20-100 $\mu$ have irregular shaped lumens. Morphologically, these vessels were regarded as venous capillaries or postcapillary venules ${ }^{5}$ though diameter was rather too large. Perivascular substance was loose, and there were many histiocytes. These facts with some evidences of fenestrations of endothelium have been compatible with rapid uptake of contrast medium or radioisotope particles and show increased permeability of the vasculature of the capsules. 


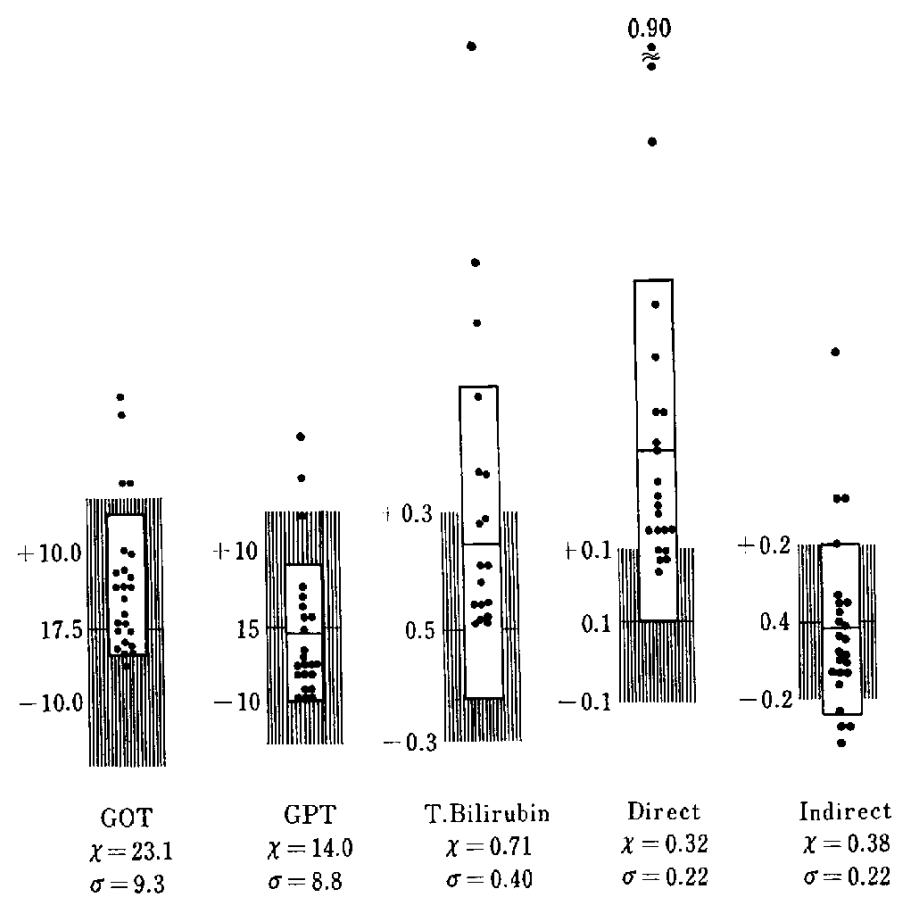

Fig. 7 Serum transaminase and serum bilirubin, for which normal range is shown by shaded area. Black points show the values of patients and frames show the mean values and standard diviations.

\section{Impairment of Liver Function}

Morphological changes of the vessels of the capsule are told in relation to the impairment of the liver function. There are so many intracranial haemorrhages in chronic liver diseases ${ }^{1)}$. Estrogen which is increased in liver disease plays some role in the change of intracranial vasculature. ${ }^{6)}$ Alcoholisms are often seen in chronic subdural hematomas. In this report heavy drinkers were $30 \%$ at least. But in the routine laboratory examinations liver functions were within normal range with some exceptional increase of GOT, GPT, bilirubin (especially direct bilirubin), and serum protein fractions, which are shown in Fig. 7 and 8 . These laboratory findings showed the tendancy to fatty liver or liver cirrhosis according to their stages of dysfunctions.

\section{Follow-Up Study}

Of 303 cases, 6 were dead in the hospitals. The follow-up study was made in 200 cases, the periods of which were more than 10 years in 68 cases, 5-9 years in 71 cases, $3-4$ years in 35 cases, and less than 2 years in 27 cases. A large number of patients spent a normal life as before the operation. There were 11 cases with limited activities and 4 cases with the help of others, but these were not sequelae of chronic subdural hematomas. The death after the discharge was 12 ; liver dysfunction 4 , gastric disorder 


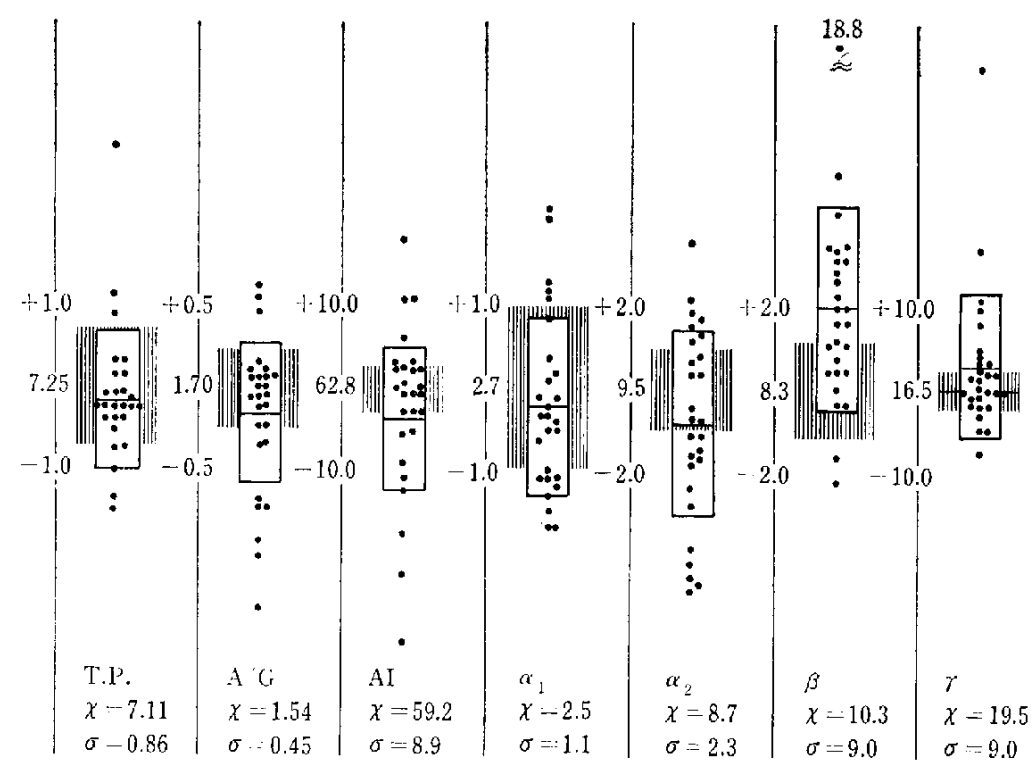

Fig. 8 Serum protein fraction. The form is the same as in Fig. 7.

Table 8 Operative method and follow-up result

\begin{tabular}{|c|c|c|c|}
\hline \multirow{2}{*}{ - . . } & \multicolumn{3}{|c|}{ operative methods } \\
\hline & $\begin{array}{c}\text { craniotomy } \\
\text { removal of capsule }\end{array}$ & $\begin{array}{l}\text { craniotomy } \\
\text { irrigation }\end{array}$ & $\begin{array}{l}\text { burr hole } \\
\text { irrigation }\end{array}$ \\
\hline & 137 cases & 33 cases & 133 cases \\
\hline re-opening & $23(17 \%)$ & $5(15 \%)$ & $15(11 \%)$ \\
\hline postoperative hematoma & $18(13 \%)$ & $1(3 \%)$ & $2(2 \%)$ \\
\hline postoperative convulsion & $21 \quad(15 \%)$ & $0(0 \%)$ & $4(3 \%)$ \\
\hline postoperative epilepsy & $11(8 \%)$ & $1(3 \%)$ & $3(2 \%)$ \\
\hline follow-up study & 92 & 22 & 89 \\
\hline normal activity & $84(91 \%)$ & $20 \quad(91 \%)$ & $84 \quad(94 \%)$ \\
\hline limited activity & $6(7 \%)$ & $0(0 \%)$ & $5(6 \%)$ \\
\hline with other's help & $2(2 \%)$ & $2(9 \%)$ & $0 \quad(0 \%)$ \\
\hline
\end{tabular}

2 , brain vascular accident 1 , pulmonary tuberculosis 1 , pneumonia 1 , trauma 1 , and unknown 2.

As for the selection of the operative method it is the problem whether or not the removal of the capsule is neccessary. Therefore operative methods, large craniotomy and removal of the hematoma with the capsule (137 cases), burr hole opening and irrigation of the hematoma without removing the capsule (133 cases), and small craniotomy and irrigation of the hematoma without removing the capsule ( 33 cases), were compared as to the postoperative complications and prognosis. The results were shown in Table 8 . In the group where the capsules were removed postoperative hematomas were seen in $13 \%$, postoperative convulsions in $15 \%$, and postoperative epilep- 
sies in $8 \%$, while in the group where the capsules were not removed, especially in burr hole opening group, they were $2 \%, 3 \%$, and $2 \%$ respectively. There were no differences of social activities among these groups in the long term follow-up results. Therefore burr hole opening and irrigation of the hematoma without removal of the capsule is superior to large craniotomy and removal of the hematoma with the capsule considering the incidence of postoperative complications. From the long term follow-up results, with exception of calcified hematoma, there were no statistical differences between the removal or non-removal of the capsule in adult type.

\section{Summary and Conclusions}

Chronic subdural hematomas were analysed statistically in 309 adult types with some comments on the dynamics of the capsule of the hematoma. On the age distribution the peak was seen in the 6th decade. $91 \%$ was male and $9 \%$ was female. History of trauma, which patients had memorized emphatically, was detected in $89 \%$. Direction of the blow exerted sagittally in $77 \%$. Trivial blow may sometimes had enough mechanical power to cause shearing effect to the brain. The role of traumas in connection with the etiology should not be underestimated. Free intervals from the trauma to the onset of the disease were seen most frequently between 1-2 months.

There were no pathognomonic signs and symptoms of the chronic subdural hematomas. Increased intracranial pressure, neurological deficit, and psychiatric manifestation were the prominent symptoms of all. Ataxic gait was apt to be a initial symptoms and was rather frequent in the bilateral hematomas which was seen in $16 \%$. One forth of total number fell into consciousness disturbance during the course. Concerning the localization hematomas were found in $88 \%$ in the frontal region and in $93 \%$ in the parietal region, which might be useful in differential diagnosis.

The shape of avascular area in the A-P view gave some keys to know the expansion and the regression of the hematomas. The content of the hematoma increases by hemorrhage from the capsule, and decreases by absorption through the capsule which was proved by using contrast medium and radioisotope. Morphological examination of the capillaries of the capsule were compatible with the increased permeability. Impairment of liver function manifested by serum protein fraction might contribute to these vascular changes.

A large number of patients have spent a normal life after the operations. The removal of the capsule of the hematoma seems unneccessary in the long term follow-up study.

\section{References}

1) Erbslöh, F.: Das ZentraInervensystem bei Leberkrankheiten. In Handbuch der speziellen pathologischen Anatomie und Histologie, XIII Nervensystem 2B. Lubarsch, O., Henke, F., \& Rössle, R. ed. Springer-Verlag, Berlin, 1958.

2) Hirakawa, K., Nakamura, N., \& Sano, K.: Spontaneous recovery of chronic subdural hematoma-Resolving subdural hematoma-r. Clinical and roentgenological considerations. Brain Nerve, 19:661-670, 1967. 
3) Nakamura, N., Hirakawa, K., \& Sano, K.: Spontaneous recovery of chronic subdural hematoma-Resolving subdural hematoma-II. Histological considerations and these backgrounds. Brain Nerve, 19:1209-1219, 1967.

4) Ommaya, A.K. \& Yarnell, P.: Subdural hematoma after whiplash injury. Lancet, $2: 237-$ 239,1969

5) Rhodin, J.A.: Ultrastructure of mammalian venous capillaries, venules, and small collecting veins. J.Ultrastruct. Res., 25: 452-500, 1968.

6) Suzuki, J.: Growing mechanism of chronic subdural hematoma. Japan-British Medical Symposium, 1972.3.1., Tokyo. 